\title{
Momentum Dependence of Single Particle Potential in Dirac Brueckner Approach
}

\author{
C.-H. Lee, T.T.S. Kuo, G.Q. Li and G.E. Brown \\ Department of Physics, State University of New York at Stony Brook, Stony Brook, NY 11794
}

\begin{abstract}
The momentum dependence of the empirical scalar and vector potentials needed for describing relativistic heavy-ion collisions at $1 \mathrm{GeV} /$ nucleon is compared with that derived from self-consistent Dirac-Brueckner calculations using the Bonn-A potential. Our calculated scalar and vector potentials exhibit a weak momentum dependence and their magnitudes decrease with nucleon momentum, in close similarity with the momentum dependence required by the empirical potentials. The effects of explicit momentum dependence on the properties of equilibrium nuclear matter are found to be small.
\end{abstract}

Typeset using REVTEX 
Heavy-ion collisions at various energies open new frontiers and provide opportunities for nuclear physics. Theoretical description of heavy-ion collisions is usually based on transport models. For heavy-ion collisions at beam energies from a few hundred $\mathrm{MeV} /$ nucleon to a few $\mathrm{GeV} /$ nucleon, the reaction dynamics is mainly governed by the stochastic two-body scattering and propagation in the mean-field potential. Transport models that include both effects, such as Boltzmann-Uehling-Uhlenbeck (BUU) [四], quantum molecular dynamics (QMD) [2], and the relativistic extensions, RBUU [3] 5] and RQMD [6], have been extensively used for the description of heavy-ion collisions in this energy region.

In the BUU or QMD calculations, the nucleon potential is usually parameterized in terms of Skyrme-type effective interaction [1,2]. The importance of the momentum dependence of the nucleon potential was realized when analysing nucleon flow and pion production in heavy-ion collisions [7, []. This has been incorporated into the BUU and QMD approaches based on various phenomenological parameterizations that are constrained by the empirical information from proton-nucleus scattering experiments [2,9, 10].

On the other hand, the relativistic transport models [3 5] are usually based on the Walecka-type effective Lagrangians [11]. In this model, nucleons feel both attractive scalar and repulsive vector potentials. The corresponding Schrödinger equivalent potential, that is to be compared with the potentials used in BUU and QMD calculations, is automatically and explicitly momentum dependent. In the mean field approximation both the scalar and vector potentials are momentum independent. The Schrödinger equivalent potential then increases linearly with nucleon kinetic energy, which is in disagreement with what has been learned from the Dirac phenomenology analysis of proton-nucleus scattering at intermediate energies [12].

In a recent paper, we have shown that the explicit momentum dependence in nucleon scalar and vector potential is important for the description of nucleon flow in heavy-ion collisions at $1 \mathrm{GeV} /$ nucleon region [13]. In that work, the momentum dependence correction was introduced by scaling the momentum independent mean field potential with a momentum dependent function extracted from the Dirac phenomenology. There have been 
similar attempts to reproduce the correct momentum dependence of the Schrödinger equivalent potential by including explicit momentum dependence in the nucleon scalar and vector potentials [14.

A main purpose of this paper is to study microscopically the origin of the momentum dependence in the scalar and vector potentials used in relativistic heavy-ion collisions [13]. The self-consitent Dirac Brueckner (DBHF) approach 15 19 is employed. It is well-known that the DBHF approach reproduces nicely the nuclear matter properties starting from realistic nucleon-nucleon interactions that are constrained by the two-nucleon data. In most DBHF calculations the scalar and vector potentials are assumed to be momentum independent. This is a good approximation for equilibrium nucleon matter, where the nucleon momenta involved are not very large. The momentum dependence was studied by ter Haar and Malfliet [16], and they found a relatively weak momentum dependence for both the real and imaginary parts of the scalar and vector potentials. Recently, Sehn et al. [20] studied the momentum dependence of the nucleon scalar and vector potentials in the first-order approximation. They first carried out the momentum independent DBHF calculation as in Ref. [17]. The scalar and vector potential so obtained were identified with a nucleon at Fermi surface, namely $|\mathbf{k}|=k_{F}$. The potentials for momenta below and above $k_{F}$ were then calculated using the formalism of Horowitz and Serot [21]. The self-consistency in the DBHF calculation has thus been neglected in Ref. [20]. We will compare our results to those of Refs. [16,20].

In this work, we follow essentially the DBHF formalism of Ref. [17]. The basic quantity is the $\tilde{G}$ matrix that satisfies the in-medium Thompson equation,

$$
\tilde{G}\left(q^{\prime}, q \mid P, \tilde{z}\right)=\tilde{V}\left(q^{\prime}, q\right)+\int \frac{d^{3} k}{(2 \pi)^{3}} \tilde{V}\left(q^{\prime}, k\right)\left(\frac{\tilde{m}(k)}{\tilde{E}(k)}\right)^{2} \frac{Q(k, P)}{2 \tilde{E}(q)-2 \tilde{E}(k)} \tilde{G}(k, q \mid P, \tilde{z}),
$$

where $\tilde{E}(k) \equiv \sqrt{\tilde{m}^{2}+(P / 2+k)^{2}}, P$ is c.m. momentum, $\tilde{z}=2 \tilde{E}(q)$ and $\tilde{m}(k)=m+U_{S}(k)$. The single-particle potential can be calculated once the $\tilde{G}$ matrix is known,

$$
\Sigma_{D B}(k)=\operatorname{Re} \int_{0}^{k_{F}} d^{3} q\left(\frac{\tilde{m}(q)}{\tilde{E}(q)}\right)\left(\frac{\tilde{m}(k)}{\tilde{E}(k)}\right)\langle k q|\tilde{G}(\tilde{z})| k q-q k\rangle .
$$


This potential is usually parameterized in terms of scalar and vector potential,

$$
\Sigma(k) \approx \frac{\tilde{m}(k)}{\tilde{E}(k)} U_{S}(k)+U_{V}(k)
$$

In Ref. [17, both the scalar and vector potentials are assumed to be momentum independent. Thus for a given density, these potentials can be determined by choosing two momenta in Eq. (3), usually $0.7 k_{F}$ and $k_{F}$. In the momentum dependent case as we consider here, we have two unknowns $\left(U_{S}\right.$ and $\left.U_{V}\right)$ for each given momentum, which cannot be uniquely determined without introducing further approximation. The customary way to do this is to assume some functional dependence on momentum for these potentials. For example, in Ref. [22], it was assumed that $U_{S, V} \approx A_{S, V}+B_{S, V} k^{2}$. Since the real part of the single particle potential vanishes at very high momentum, we expect the following parameterization to be more appropriate

$$
U_{S, V}(k)=\frac{\left(U_{S, V}\right)_{0}}{1+\alpha_{S, V}\left(k / k_{F}\right)^{\beta_{S, V}}}
$$

Actually, in Ref. [13], this kind of functional dependence was found to fit the Dirac phenomenology rather well. One of the purposes of this work is thus to study whether this kind of simple parameterization is supported by microscopic DBHF calculation. Numerically we find that it is rather difficult to determine the six parameters altogether in the minimization procedure. We have checked the variation of $\beta_{S, V}$ around 0.5 , and find that $\beta_{S, V} \approx 0.5$ provides a smooth self-consistent procedure, and the final results are not very sensitive to some small change in $\beta_{S, V}$. The remaining four parameters are then determined by a minimization procedure,

$$
\chi^{2}=\int_{0}^{k_{F}} d^{3} k\left(\Sigma_{D B}(k)-\Sigma(k)\right)^{2}
$$

Our self-consistent procedure is the following. For a given density as specified by Fermi momentum $k_{F}$, we choose reasonable initial values for $\left(U_{S, V}\right)_{0}$ and $\alpha_{S, V}$. These, together with Eq. (4) provide $U_{S, V}$ for all momenta. These potentials are then used as inputs in Eq. (1) to determine the $\tilde{G}$ matrix, from which we obtain the single-particle potential $\Sigma_{D B}$ using Eq. 
(2). Our new set of $\left(U_{S, V}\right)_{0}$ and $\alpha_{S, V}$ can then be determined by minimizing Eq. (5) against $\Sigma_{D B}$. This procedure is continued until desired self-consistency is achieved. The quality of our self-consistency procedure can be seen in Fig. [ [ where we compare the single-particle potential from the DBHF calculation (solid symbols) and from Eqs. (3) and (4). There are slight deviations near $k=0$ because in our definition of $\chi^{2}$, Eq. (5), the contributions from small momenta are suppressed. The self consistent parameters are summarized in Tab. [1].

In Fig. 2 we show our results for the momentum dependence of the scalar and vector potentials at normal nuclear matter density. Both are seen to decrease in magnitude as nucleon momentum increases. Also shown in the figure are the results of ter Haar and Malfliet (HM) [16] and those of Sehn et al. (SFF) [20]. Our results are very close to those of ter Haar and Malfliet, which supports our parametrization functional, Eq. (4). The results of Sehn et al. show a much stronger momentum dependence than ours and those of ter Haar and Malfliet [16], because of the lack of the self-consistency. We show in Fig. 3 the momentum dependence of the nucleon scalar and vector potentials at several different densities.

From the scalar and vector potential we can calculated the Schrödinger equivalent potential given by

$$
U_{s c h}(k)=U_{S}(k)+\left(\frac{E_{k i n}(k)}{m}+1\right) U_{V}(k)+\frac{U_{S}(k)^{2}-U_{V}(k)^{2}}{2 m},
$$

where $E_{k i n}$ is the nucleon kinetic energy. The results are shown in Fig. $\$$. The solid symbols are the Schrödinger equivalent potential extracted from the Dirac phenomenology analysis [12]. The solid and dashed curves are obtained with and without explicit momentum dependence in the scalar and vector potentials. The dotted is from ref. [16]. It is seen that the explicit momentum dependence in the scalar and vector potentials improves, to some extent, the agreement with the Dirac phenomenology. The Schrödinger equivalent potential from our self-consistent calculation still show stronger kinetic energy dependence than that of the Dirac phenomenology. The onset of inelastic processes at high beam energies might explain part of this discrepancy. 
It is also of interest to see how the nuclear equation of state are affected by the explicit momentum dependence.

$$
E / A=\frac{1}{\int_{0}^{k_{F}} d^{3} k} \int_{0}^{k_{F}} d^{3} k\left(\frac{M \tilde{M}(k)+k^{2}}{\tilde{E}(k)}+\frac{1}{2} \Sigma(k)\right)-M .
$$

In Fig.5, the binding energies are plotted as a function of density. The solid and dashed curves are obtained with and without explicit momentum dependence in the scalar and vector potentials. It is seen that the effects of the explicit momentum dependence on equilibriu nuclear matter is insignificant. The saturation density in both cases are about $\sim 0.18 \mathrm{fm}^{-3}$. The binding energy decreases by about $0.2 \mathrm{MeV}$ when explicit momentum dependence is included.

In summary, we studied the momentum dependence of nuclear scalar and vector potentials self-consistently in the formalism of Dirac Brueckner approach. The magnitudes of both potentials have been found to decrease slowly with increasing nucleon momentum or kinetic energy. This improves, to some extent, the agreement with the Dirac phenomenology. The effects of explicit momentum dependence on the properties of equilibrium nuclear matter were found to be small.

\section{ACKNOWLEDGEMENT}

We would like to thank M. Prakash, M. Rho and C.M. Ko for helpful discussions. We also appreciate R. Machleidt for sending us the code of the relativistic Bonn potential and helpful discussions. This work was supported by the U.S. Department of Energy under grant no. DE-FG02-88ER40388. The work of CHL was supported in part by Korea Science and Engineering Foundation. 


\section{REFERENCES}

[1] G. F. Bertsch and S. Das Gupta, Phys. Rep. 160 (1988) 189.

[2] J. Aichelin, Phys. Rep. 202 (1991) 235.

[3] W. Cassing, V. Metag, U. Mosel, and K. Niita, Phys. Rep. 188 (1990) 363.

[4] G. Q. Li, C. M. Ko, X. S. Fang, and Y. M. Zheng, Phys. Rev. C 49 (1994) 1139.

[5] C. M. Ko and G. Q. Li, J. Phys. G 22 (1996) 1673.

[6] H. Sorge, H. Stöcker, and W. Greiner, Ann. Phys. 192 (1989) 266.

[7] C. Gale, G. Bertsch, and S. Das Gupta, Phys. Rev. C 35 (1987) 1666.

[8] J. Aichelin, A. Rosenhauer, G. Peilert, H. Stöcker, and W. Greiner, Phys. Rev. Lett. 58 (1987) 1926.

[9] M. Prakash, T.T.S. Kuo and S.D. Gupta, Phys. Rev. C 37 (1988) 2253.

[10] C. Gale, G.M. Welke, M. Prakash, S.J. Lee and S.D. Gupta, Phys. Rev. C 41 (1990) 1545.

[11] B. D. Serot and J. D. Walecka, Adv. Nucl. Phys. 16 (1986) 1.

[12] E. D. Cooper, S. Hama, B. C. Clark, and R. L. Mercer, Phys. Rev. C 47 (1993) 297.

[13] G. Q. Li, G. E. Brown, C.-H. Lee, and C. M. Ko, to be published.

[14] T. Maruyama, W. Cassing, U. Mosel, S. Teis, and K. Weber, Nucl. Phys. A 573 (1994) 653.

[15] M. R. Anastasio, L. S. Celenza, W. S. Pong, and C. M. Shakin, Phys. Rep. 100 (1983) 327.

[16] B. ter Haar and R. Malfliet, Phys. Rep. 149 (1987) 207.

[17] R. Machleidt, Adv. Nucl. Phys. 19 (1989) 189. 
[18] R. Brockmann and R. Machleidt, Phys. Rev. C 42 (1990) 1965.

[19] G. Q. Li, R. Machleidt, and R. Brockmann, Phys. Rev. C 45 (1992) 2782.

[20] L. Sehn, C. Fuchs and Amand Faessler, nucl-th/9701060, "Nucleon self-energy in the relativistic Brueckner approach".

[21] C.J. Horowitz and B.D. Serot, Nucl. Phys. A464 (1987) 613.

[22] H. Müther, M. Prakash, and T.L. Ainsworth, Phys. Lett. B 199 (1987) 469. 


\section{TABLES}

\begin{tabular}{|c||c|c|c|c|}
\hline$u\left(\rho / \rho_{0}\right)$ & $U_{s 0}(\mathrm{MeV})$ & $\alpha_{s}$ & $U_{v 0}(\mathrm{MeV})$ & $\alpha_{v}$ \\
\hline 0.70 & -426.2505 & 0.6178 & 370.6928 & 0.8754 \\
0.85 & -474.3686 & 0.4283 & 408.2838 & 0.5805 \\
1.00 & -494.2272 & 0.3426 & 420.5226 & 0.4585 \\
1.12 & -499.1539 & 0.2790 & 420.0603 & 0.3687 \\
1.24 & -518.8160 & 0.2566 & 436.9299 & 0.3361 \\
1.37 & -537.0683 & 0.2357 & 453.5746 & 0.3050 \\
1.51 & -586.0774 & 0.2652 & 508.8679 & 0.3515 \\
\hline
\end{tabular}

TABLE I. Self-consistent parameters with $\rho_{0}=0.166 \mathrm{fm}^{-3}$. 


\section{FIGURES}

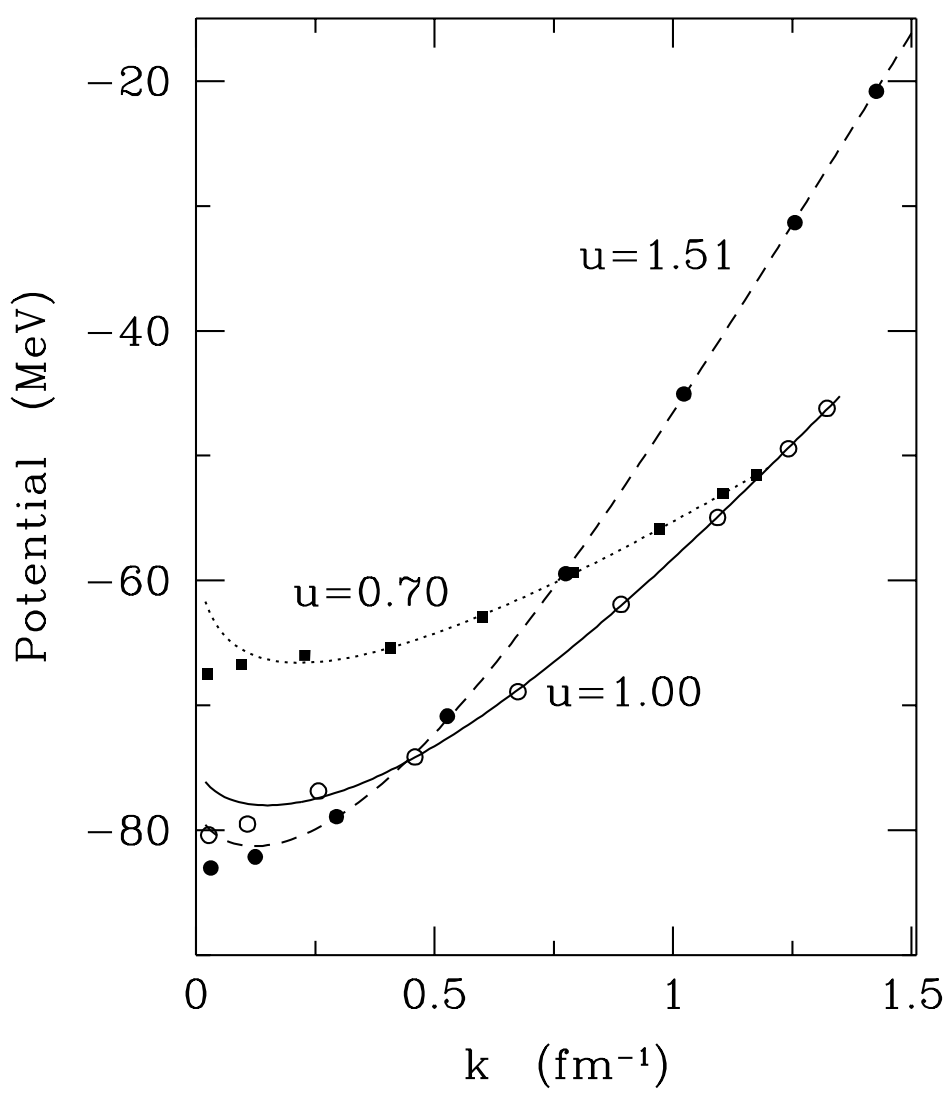

FIG. 1. Momentum dependence of single particle potential. The solid symbols are the results of DBHF calculation and the lines are from Eqs. (3) and (4). 


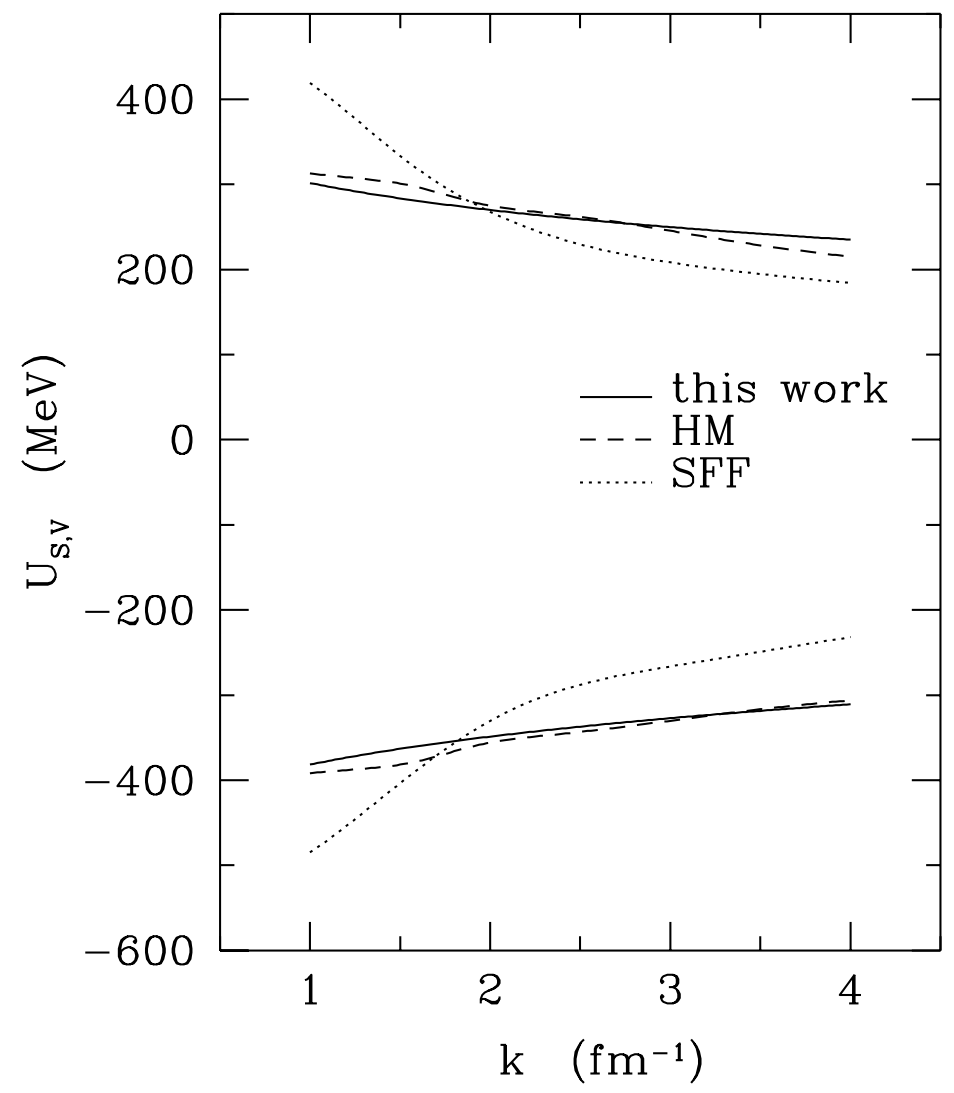

FIG. 2. nucleon scalar and vector potential as a function of nucleon momentum at normal nuclear matter density. The solid curves are our results, while the dashed and dotted curve are the results from Refs. [16] (HM) and [20] (SFF), respectively. 


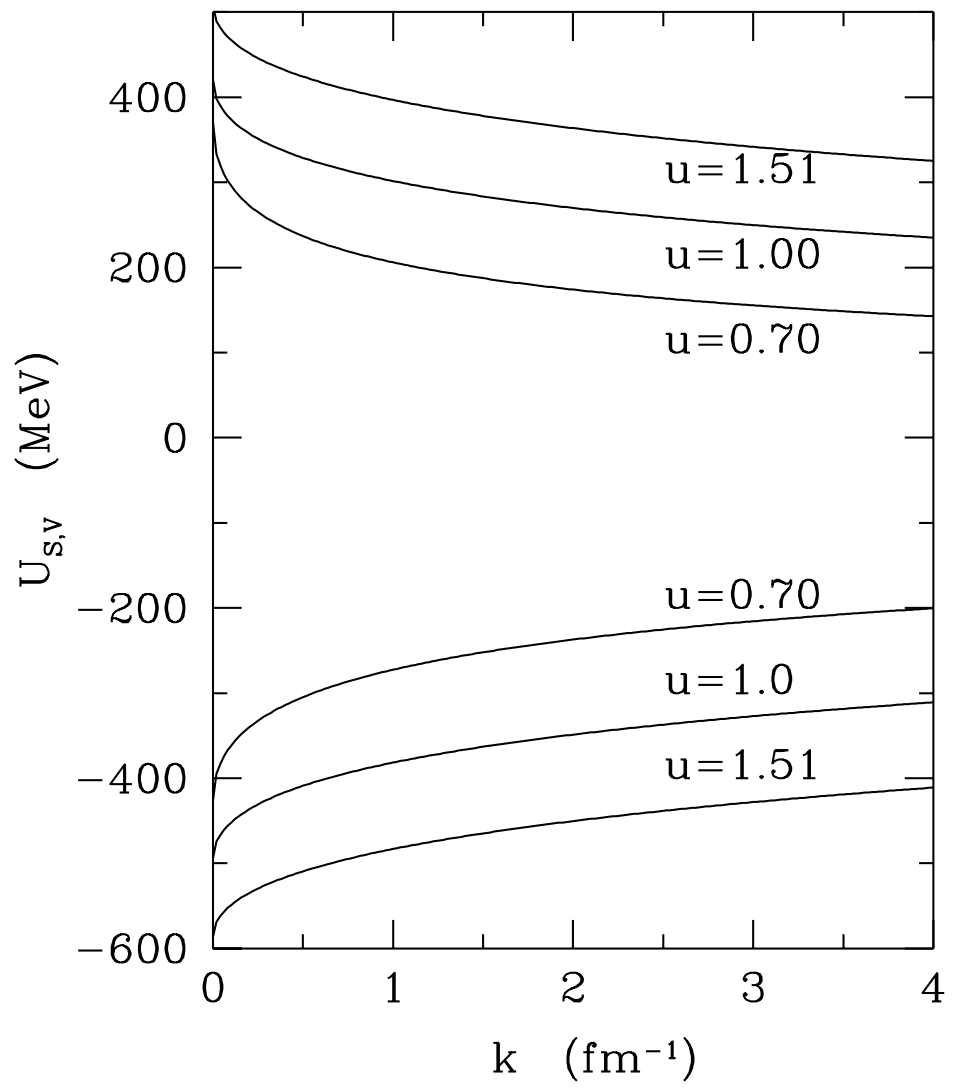

FIG. 3. nucleon scalar and vector potential as a function of nucleon momentum at several different densities. 


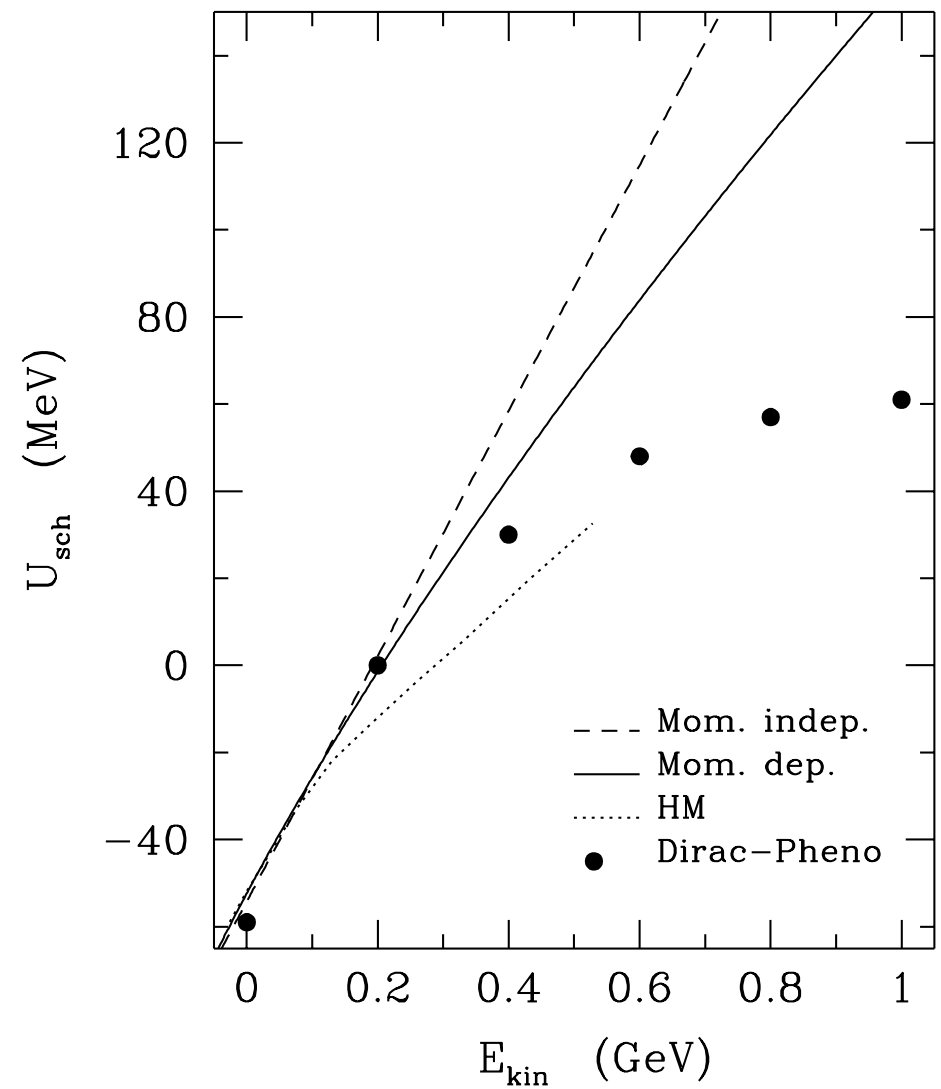

FIG. 4. Schrödinger equivalent potential in the DBHF calculation with and without explicit momentum dependence. The solid circles are extracted from the Dirac phenomenology analysis [12. The results of Haar and Malfliet (HM) [16] are plotted in dotted line. 


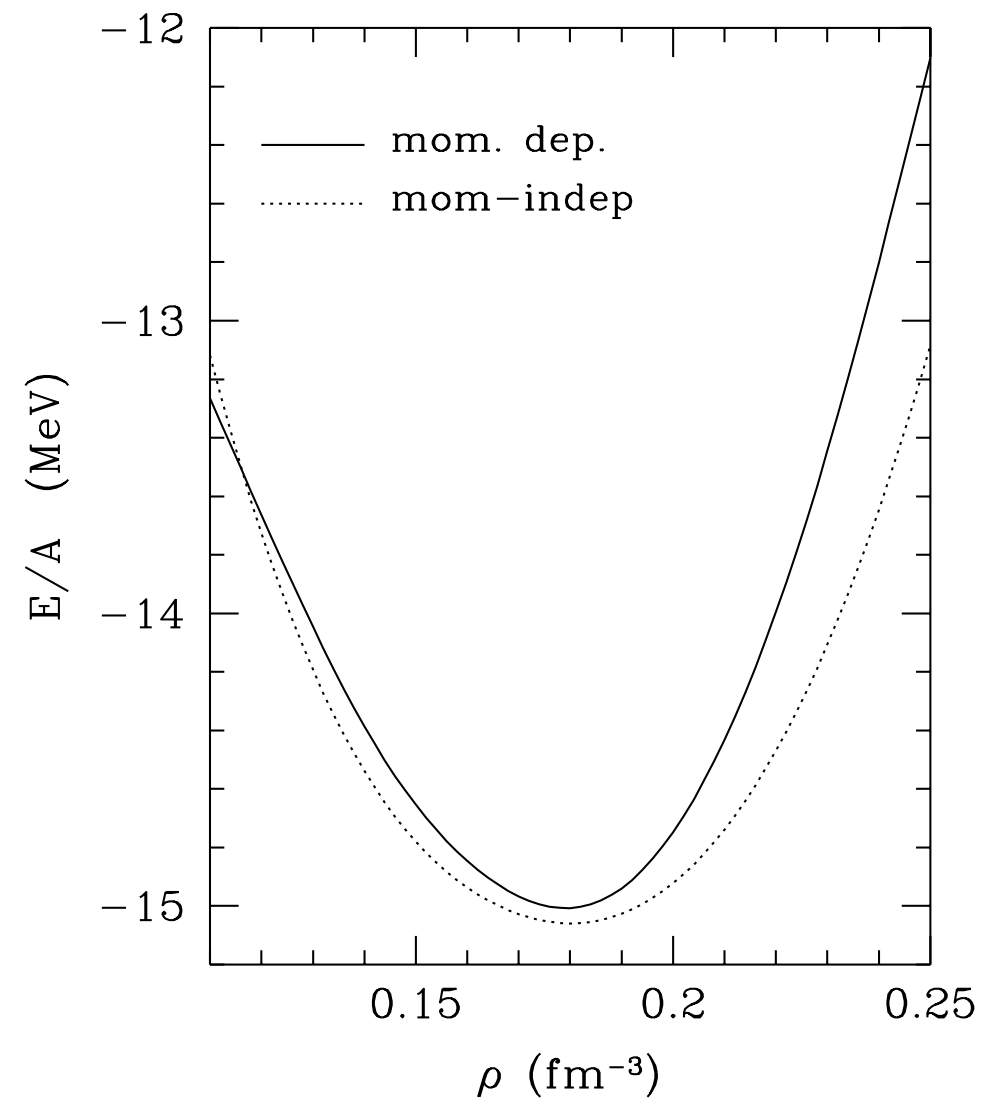

FIG. 5. Binding energies in the DBHF calculation with and without explicit momentum dependence. 